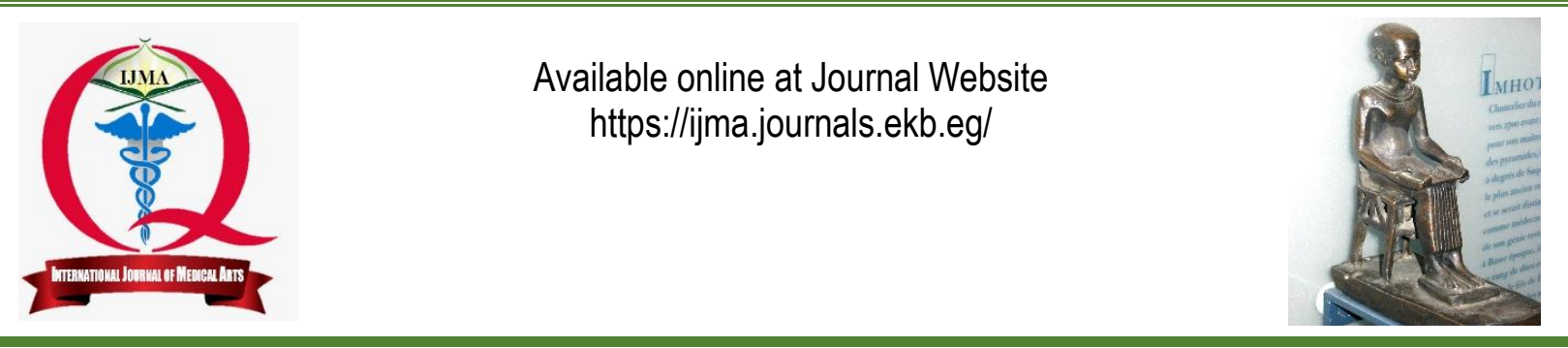

Original article

\title{
Correlation between Diabetic Retinopathy and the Severity of Coronary Artery Disease Determined by Coronary Angiography in Patients with Type II Diabetes Mellitus
}

Mohammed Adel Attiaa; Mohammed Ibrahim Elraghya; Akram Fekry Elgazarb; Sherif Ragab Nayela

Department of Cardiology, Damietta Faculty of Medicine, Al-Azhar University, Egypt[a].

Department of Ophthalmology, Damietta Faculty of Medicine, Al-Azhar University, Egypt[b].

Corresponding author: Mohammed Ibrahim Elraghy

Email: mohamedelraghy2@azhar.edu.eg

Received at: October 1, Revised at: October 30, 2019; Accepted at: November 25, 2019; Available online at: December 2, 2019 DOI: 10.21608/ijma.2019.63391

\section{ABSTRACT}

Background: Diabetes mellitus [DM] is one of the most widespread chronic diseases. It is commonly known by its serious vascular complications including micro-vascular [as retinopathy] and macro-vascular [as ischemic heart diseases] complications. So, a correlation between those complications may be found.

Aim of the work: To investigate the correlation between diabetic retinopathy and increased risk of coronary artery diseases.

Patients and methods: This study enrolled fifty patients with type 2 diabetes mellitus [DM] who were referred for coronary angiography. Full History, clinical examination, biochemical tests, electrocardiography [ECG], Echocardiography, coronary angiography and fundus examination were carried out. Diabetic retinopathy was detected and classified; patients were classified into two groups: a group [A] with diabetic retinopathy [DR] and a group [B] without diabetic retinopathy. Coronary diseases were assisted by coronary angiography using two different scores.

Results: Population characters, including age, risk factors, duration of DM, lipid profile, angiography scores, were comparable between the two study groups. Patients in group $[A]$ had significantly higher number of diseased vessels and higher Gensini score than those of Group [B]. The correlation was significant between the presence of the $D R$ and both the number of diseased vessels $[r=0.532 P<0.001]$ and Gensini score $[r=0.881, P<0.001]$.

Conclusion: The diabetic retinopathy is a serious risk factor for increasing the severity of coronary artery diseases and can consider as a predictor of $\mathrm{CHD}$ in patients with $\mathrm{DR}$.

Keywords: Coronary artery disease; Diabetes mellitus; Diabetic retinopathy; Coronary angiography; Prediction.

This is an open access article under the Creative Commons license [CC BY] [https://creativecommons.org/licenses/by/2.0/]

Please cite this article as: Attia MA, Elraghy MI, Elgazar AF, Nayel SR. Correlation Between Diabetic Retinopathy and The Severity of Coronary Artery Disease Determined by Coronary Angiography in Patients with Type II Diabetes Mellitus. IJMA 2020; 2[1]: 204-210. 


\section{INTRODUCTION}

Cardiovascular diseases [CVD] are believed to be one of the most leading causes of death in diabetic patients[ ${ }^{[1]}$. However, many diabetic patients with coronary artery disease [CAD] don't have any other classic risk factor for coronary artery disease and half of them have a normal lipid profile[2].

Although diabetes was once deemed as a risk for CAD risk, heterogeneity of such risk was also increasingly recognized, making the new guidelines looking for a new assessment tool to specifically assess the risk for diabetic patients[3,4].

There is still a crucial need to discover a CAD prediction algorithm for patients with diabetes as the Framingham risk scores did not perform desirably in this population [5,6], and existing CAD models for prediction in diabetic patients lack accuracy[].

Diabetic retinopathy [DR] is also a well-Known micro-angiopathic complication of DM with marked association with cardiovascular risk factors. It is usually detected by retinal [fundus] photographs. There are many qualitative markers for microvascular pathology assessment. However, now, computer-assisted programs can enable us to detect the micro-vascular damage [as retinal vascular caliber] ${ }^{[8] \text {. }}$

A marked association has appeared between those measures of microvascular damage and CAD not only in the general population but also in cohorts with diabetes, suggesting potential reflection of that retinal measures captured from retinal photographs, reflecting generalized microvascular disease, on further refining of CAD risk in diabetics ${ }^{[9]}$..

Recent studies had suggested that retinopathy is considered an early sign of other vascular complications, being highly associated with development of $C A D^{[10]}$.

This is propped by previous studies that linked retinopathy signs with more ischemic T-wave changes on electro-cardiogram, coronary perfusion defects, poorer coronary flow reserve and lower coronary collateral scores, than those without retinopathy[11].

On the one hand, ophthalmologists focus mainly on the diagnosis and treatment of $\mathrm{DR}$, but usually ignore the importance of these risk factors in the assessment of CVD; while the physicians often pay too much attention to $C A D$, but usually ignore the severity of DR. This way, the complicated conditions of diabetic patients are often overlooked and can significantly affect their quality of life. So about questioning, if screening for $\mathrm{CHD}$ should be applied in asymptomatic DR patients. Till now, neither consensus nor guideline has been established.

\section{AIM OF THE WORK}

We aimed here to investigate the correlation between presence and grading of DR, and the severity and extent of CAD in patients with type II DM.

\section{PATIENTS AND METHODS}

Fifty cases were selected from consecutive typeII DM patients who had been admitted for coronary angiography because of suspected CAD to the catheterization laboratory of Al-Azhar University Hospitals between May 2018 and May 2019. TypeII DM was diagnosed as stated in the by the American Diabetes Association [ADS] ${ }^{12]}$.

We exclude patients who have a duration of DM of less than one year, type-I diabetes mellitus, uncontrolled hypertension [systolic blood pressure $>180 \mathrm{~mm} \mathrm{Hg}$ and/or diastolic blood pressure > 100 $\mathrm{mmHg}$, previous coronary bypass surgery, known non-diabetic retinal disease and patients who have acute coronary syndrome. This study has been approved by the local Institutional Review Board.

After the informed consent was taken from all patients, all of them underwent full history taking, general and local examination, biochemical tests including: complete blood count [CBC], glycosylated hemoglobin [HgbA1c], serum creatinine, total cholesterol [TC], triglycerides [TG], low-density lipoprotein cholesterol [LDL-C] and high-density lipoprotein cholesterol [HDL-C]. Standard 12-lead surface Electrocardiogram [ECG] and Echocardiographic assessment.

Detection Ophthalmoscopy by Optical coherence tomography [OCT] was performed to all patients by an experienced ophthalmologist. The presence of retinopathy was detected and classified based on the International Clinical Diabetic 
Retinopathy Scale into two groups, group $A$, with diabetic retinopathy and group $B$, without diabetic retinopathy.

Selective coronary angiography had been applied from the femoral approach using Judkin's technique. By the usage of cine-angiographic equipment multiple views had been taken, with left anterior descending and left circumflex coronary arteries were visualized in at least four views and the right coronary artery was visualized in at least two views [12]

Scores were calculated from coronary angiograms by two techniques:

1] Vessel score, which is the vessel number with significant stenosis [the reduction in $50 \%$ or more in the lumen diameter]. The range of the scores was 0 to 3 , and it depended on the vessels involving. Left main artery stenosis had been considered a single vessel disease.

2] Severity score: It's knows as the Gensini score, which was described previously. In the previously mentioned Gensini score, give a 1 for $25 \%$ stenosis and 2 for $50 \%, 4$ for $75 \%, 8$ for $90 \%$, 16 for $99 \%$ and 32 for the total occlusion. Then the scores were multiplied by a factor ${ }^{[13]}$.

The sum of all sector scores hands out the Gensini score, which emphasizes the degree of disease severity. These scores had been determined through one of the investigators without any learning about the clinical history or retina results.

\section{Statistical analysis:}

Quantitative data were expressed in mean \pm standard deviation [SD] to measure the central tendency and dispersion of quantitative data.

Qualitative data were expressed in numbers and percentages. Correlation coefficient [r] was used to detect the correlation between different variables. The level of significance was taken at $P$ value < 0.05 . Statistical analysis was completed using SPSS [Statistical Package for Social Science version 22]

\section{RESULTS}

This study included fifty patients with type-II DM defined according to American Diabetes Association ${ }^{[12]}$ and suspected CAD who underwent elective coronary angiography, 23 of them [46\%] were males, while 27 [54\%] were females; 36 patients [72\%] were hypertensive, 17 patients [34\%] were dyslipidemic, 19 patients [38\%] were smokers, [9] with previous family history [18\%] and 13 has ischemic heart disease [IHD] [26\%].

In table [1]; patients in group [A] were older, with longer duration of $\mathrm{DM}$, had higher levels of $\mathrm{HbA} 1 \mathrm{c}$ and had higher in lipid profile levels than patients in group B. There was no significant difference between the two groups regarding, age, and Risk factors for $C A D$ and serum creatinine.

Among group $[A]$, all patients had evidence of significant CAD by coronary angiography, 18 [72\%] of them had multi-vessel disease, 6 [24\%] had 2vessel disease and only one [4\%] patient had single vessel disease. On the other hand, among group [B], 9 [36\%] patients had angiographic evidence of significant CAD; of them 2 [8\%] patients had multivessel disease, 2 [8\%] patients had 2-vessel disease and 5 [20\%] patients had single vessel disease.

By Comparison of coronary angiography scores between the two groups, patients in the group $[A]$, had a significantly higher vessel number $[0.64 \pm 0.95$ versus $2.36 \pm 1.03, P<0.001]$, and Gensini Score [20.72 \pm 31.95 versus 68.72 $\pm 29.95, P<0.001]$.

As shown in Table [2], there was significant increase of vessels score and Gensini score in group A when compared to group B $[2.68 \pm 0.55$, $68.72 \pm 29.95$ vs $0.64 \pm 0.95, \quad 20.72 \pm 31.95$ respectively].

In addition, there was a significant positive correlation between the presence of $\mathrm{DR}$ and number of diseased vessels [ $r=0.532, P=0.001$ ]. There was a highly significant positive correlation between the presence of DR and Gensini score $[r=$ $0.881, P<0.001$ ]. 
Table [1]: Baseline characters of the Study groups

\begin{tabular}{|c|c|c|c|c|}
\hline & Group A & Group B & P-value \\
\hline \multirow[t]{5}{*}{ Age } & $40-$ & $1[4.0 \%]$ & $6[24 \%]$ & \multirow{5}{*}{$0.38[\mathrm{NS}]$} \\
\hline & $50-$ & $7[28.0 \%]$ & $9[36.0 \%]$ & \\
\hline & $60-$ & $15[60.0 \%]$ & $9[36.0 \%]$ & \\
\hline & $70-72$ & $2[8.0 \%]$ & $1[4.0 \%]$ & \\
\hline & Mean $\pm S D ;$ Mini.-Maxi. & $59.16 \pm 6.83 ; 47-70$ & $57.3 \pm 7.60 ; 45-72$ & \\
\hline \multirow[t]{2}{*}{ Sex } & Male & $11[44.0 \%]$ & $12[48.0 \%]$ & \multirow[t]{2}{*}{0.7 [NS] } \\
\hline & Female & $14[56.0 \%]$ & $13[52.0 \%]$ & \\
\hline \multirow{3}{*}{$\begin{array}{l}\text { Duration of } \\
\text { DM }\end{array}$} & $10-15$ & $7[28 \%]$ & $12[48 \%]$ & \multirow{3}{*}{$0.05 *$} \\
\hline & $16-20$ & $4[14.0 \%]$ & $5[20.0 \%]$ & \\
\hline & $>20$ & $14[56.0 \%]$ & $8[32.0 \%]$ & \\
\hline \multirow[t]{5}{*}{ Risk factors } & Hypertension & $19[76 \%]$ & $17[68 \%]$ & $0.5[\mathrm{NS}]$ \\
\hline & Dyslipidemia & $8[32 \%]$ & $9[36 \%]$ & $0.7[\mathrm{NS}]$ \\
\hline & Smoking & $10[40 \%]$ & $9[36 \%]$ & $0.7[\mathrm{NS}]$ \\
\hline & Family history & $4[16 \%]$ & $5[20 \%]$ & $0.7[\mathrm{NS}]$ \\
\hline & $\mathrm{IHD}$ & $8[32 \%]$ & $5[20 \%]$ & $0.3[\mathrm{NS}]$ \\
\hline \multicolumn{2}{|l|}{ HA1C } & $8.44 \pm 1.39 ; 6.40-11.30$ & $7.05 \pm 0.75 ; 5.8-8.30$ & $0.005^{*}$ \\
\hline \multicolumn{2}{|c|}{ Cholesterol } & $219.9 \pm 63.9 ; 112.0-312.20$ & $177.9 \pm 56.9 ; 104.9-306.5$ & $0.01^{*}$ \\
\hline \multicolumn{2}{|c|}{ Serum creatinine } & $0.96 \pm 0.18 ; 0.6-1.4$ & $0.94 \pm 0.22 ; 0.6-1.4$ & $0.7[\mathrm{NS}]$ \\
\hline
\end{tabular}

NS: non-significant; *: Significant

Table [2]: Comparison between groups A and B as regard vessel and Gensini Score

\begin{tabular}{|l|l|l|c|}
\hline & \multicolumn{1}{|c|}{ Group A } & \multicolumn{1}{|c|}{ Group B } & P-value \\
\hline Vessel Score & $2.68 \pm 0.55 ; 1-3$ & $0.64 \pm 0.95 ; 0-3$ & $<0.0001^{*}$ \\
\hline Gensini Score & $68.72 \pm 29.95 ; 15-138$ & $20.72 \pm 31.95 ; 0-100$ & $<0.0001^{*}$ \\
\hline
\end{tabular}

NS: non-significant; *: Significant

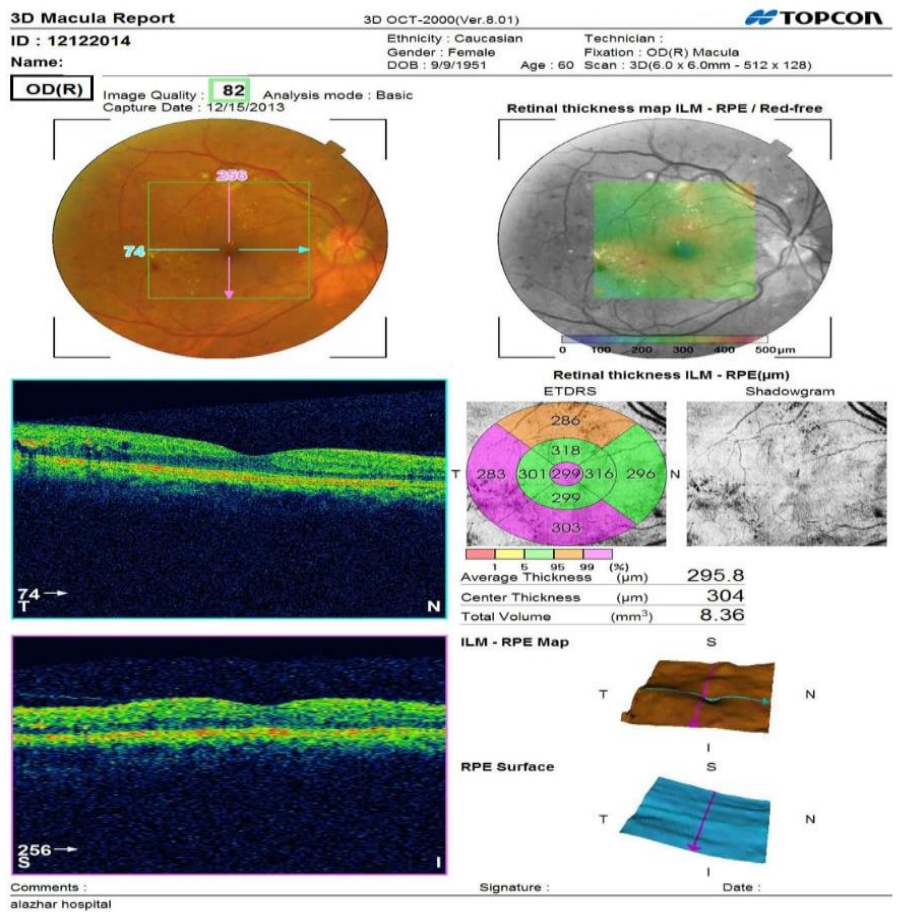

Figure [1]: OCT showing moderate non-proliferative diabetic retinopathy 


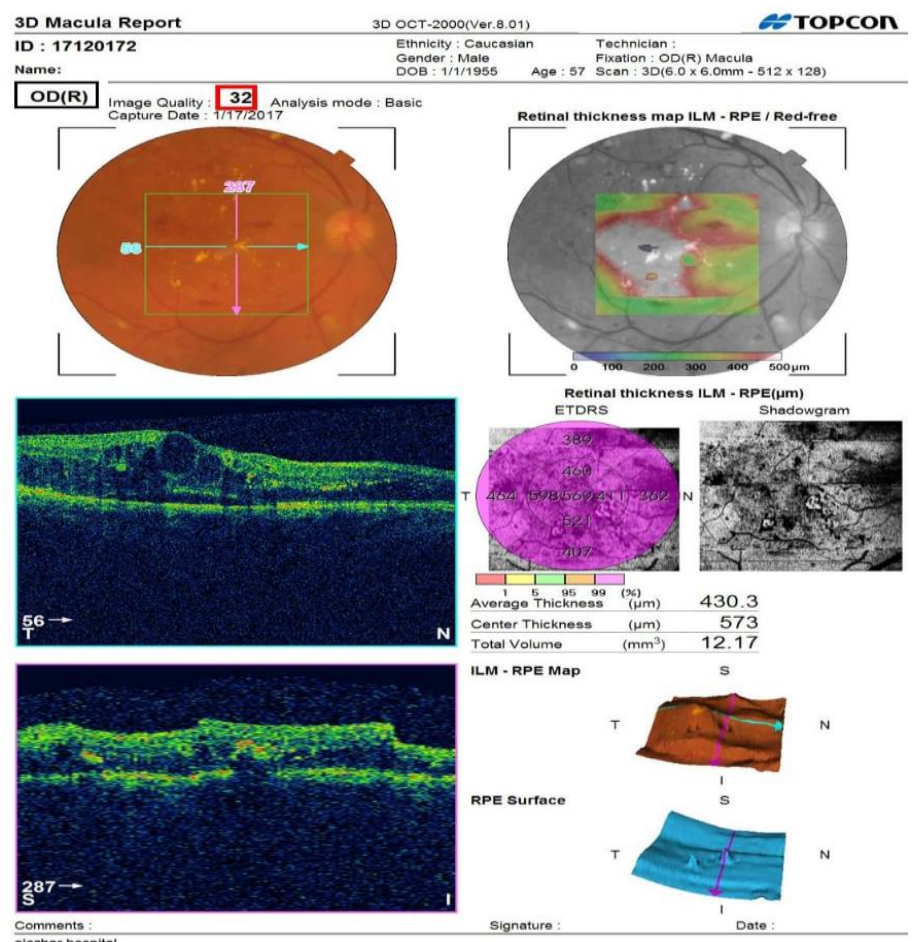

Figure [2]: OCT showing severe non-proliferative diabetic retinopathy

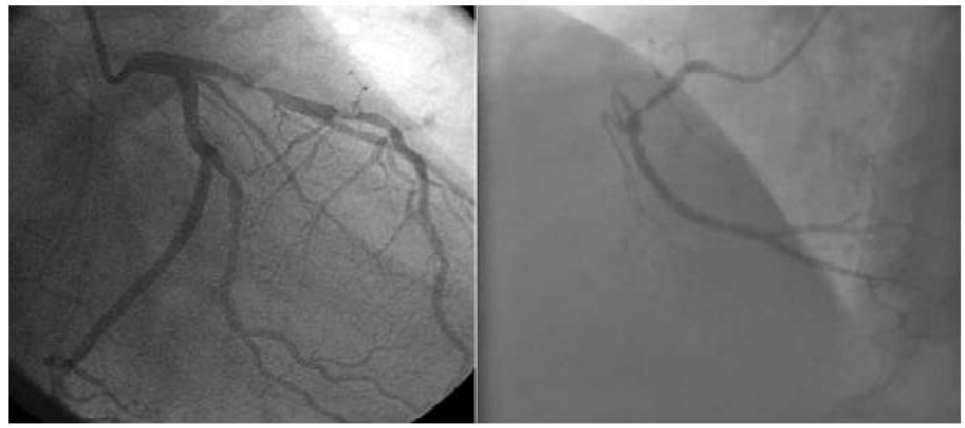

Figure [3]: Coronary angiogram showing multiple significant coronary stenosis

\section{DISCUSSION}

Cardiovascular diseases are considered to be to be main cause of death for patients with type-2 diabetic patients. It accounts for about 3 times as many deaths among diabetic as among nondiabetics. DR was found to be correlated with CAD risk of and to be a predictive tool for CAD events in diabetic patients ${ }^{[]]}$. Similarly, with patients have cardiovascular diseases, diabetes carries a huge risk of worse outcomes and increases the morbidity and mortality risk, especially when associated complications as nephropathy, retinopathy and possibly neuropathy are present ${ }^{[13]}$.

DR is a serious common micro-vascular complication in diabetic patients. It occurs in about $19 \%-30 \%$ e of adult diabetic patients ${ }^{[6]}$.
In our research, we investigated the relation between DR and severity of CAD. We used coronary angiographic scores [Gensini and vessel scores] to examine the CAD severity.

Our study results demonstrated a relationship between the severity of $D R$ and the severity of $C A D$ detected by coronary angiography.

Our results were agreed with results from Um et al.[10]. They reviewed 175 sheets of patients records retrospectively, patients were classified into three groups according to the severity of DR, 38 patients had DR, and 88 non- proliferative $D R$, and 49 with proliferative DR. Among those 3 diabetic retinopathy grading groups, there were significant differences in the duration of diabetes $[p<0.001]$ and their mean $\mathrm{HbA} 1 \mathrm{c}$ level [p=0.003], and no other significant differences. Comparing DSCT findings to 
diabetic retinopathy severity, there was a significant increase in the ACS. [p<0.001], as well as in the number of significant stenotic vessels [ $p=0.011$, as the diabetic retinopathy severity increased.

However our study differs from this one in that they did not determine severity of retinopathy and our group A was slightly older in age.

Our results were also consistent with a study by Cheung et al. ${ }^{[14]}$ who conducted a populationbased, prospective cohort study consisting of 1,524 middle-aged individuals with type 2 diabetes without prevalent CAD and stroke. The signs of DR were graded and CAD events [myocardial infarction, fatal $C A D$, or coronary revascularization] were identified. 214 [14.7\%] patients had diabetic retinopathy. after follow-up of 7.8 years, there were 209 [13.7\%] develop CAD events. The presence of DR was associated with a 2 fold higher risk of devolving CAD events 2.07 [95\% ci $1.38-3.11]$ ] and a 3 folds higher risk of fatal CAD [3.35 [1.40-8.01]].

Tian et al. ${ }^{[15]}$ reported that, increased risk of $C A D$ was significant in both men [1.89 [1.08-3.31]] and women [2.16 [1.16-4.02]] with diabetic retinopathy. This association independent of the glycemic level.

The major difference between our study and the previous two studies, that we evaluated the severity of CAD by coronary angiography, which is the gold standard tool for diagnosis of CAD.

Our findings are also consistent with previous epidemiological studies, reported that patients have DR have a higher incidence to develop cardiovascular disease ${ }^{[3,4]}$.

In the present study; old age, longer duration of DM were significantly evident in the stenotic group, Tian et al. [15] reported that these factors have no clinical significance on the process of atherosclerosis.

Also in our study, there are significant differences in cholesterol level in the group with diabetic retinopathy, although in Yang et al. [16], no significant association was found between blood lipid parameters and diabetic retinopathy. The findings in Koch et al. ${ }^{[17]}$ showed that, the CVD risk factors even the clinical symptoms, are not considered a valid tool for prediction of cardio- vascular $[\mathrm{CV}]$ events and that coronary angiography should be performed in all diabetic patients before renal transplantation. Williams et al. found combined micro-vascular and macrovascular sequel in $24 \%$ of diabetics [18].

Coronary angiography is the absolute method and the cornerstone for diagnosing $C A D$, although not always handy in many cardiovascular units in the developing countries ${ }^{[18]}$.

Diagnosis of diabetic retinopathy is an easy and available outpatient clinical procedure. Its presence is a call for a complete examination the risk factors of CVD besides echocardiography stress ECG and coronary angiography[2].

Cheung et al. [14] had also reported similar results saying that $D R$ is considered as an independent risk marker for CAD. Moreover, Bailey and Sparrow found that $24 \%$ of diabetic patients that have retinopathy have angina and myocardial infarction[19].

\section{Study limitation}

First, the small sample size that may reduce its generalizability. Also, the coronary CT angiography has become more popular, but only a small numbers of patients with diabetes who decide to perform this procedure, moreover it is rare for those with no symptoms to do. Besides, not all diabetic patients are given a retinal evaluation. Therefore, our patient number was small and a multi-centered prospective study is needed in the future.

Conclusion: A strong relation was found between presence of diabetic retinopathy and its severity with the severity of CAD in patients with type-II DM and count to be predictor tool for CAD in the future.

\section{Conflict of interest}

No conflict of interest.

\section{REFERENCES}

1-Gu K, Cowie CC, Harris MI. Diabetes and decline in heart disease mortality in US adults. JAMA. 1999; 281[14]:1291-1297 [DOI: 10.1001/ jama. 281. 14.1291].

2. Wannamethee SG, Shaper AG, Whincup PH, Lennon L, Sattar N. Impact of diabetes on cardiovascular disease risk and all-cause mortality in older men: 
Influence of age at onset, diabetes duration, and established and novel risk factors. Arch Intern Med. 2011; 171[5]:404-410. [Doi: 10.1001/ archinternmed. 2011.2].

3. Gore MO, McGuire DK, Lingvay I, Rosenstock J. Predicting Cardiovascular Risk in Type 2 Diabetes: the Heterogeneity Challenges. Curr Cardiol Rep. 2015; 17[7]. [DOI: 10.1007/s11886-015-0607-7].

4. Buse JB, Ginsberg HN, Bakris GL, Clark NG, Costa F, Eckel $\mathbf{R}$, et al. Primary prevention of cardiovascular diseases in people with diabetes mellitus: A scientific statement from the American Heart Association and the American Diabetes Association. Circulation. 2007; 115[1]:114-126. [DOI: 10.1161/ CIRCULATIONAHA. 106.179294].

5. Guzder RN, Gatling W, Mullee MA, Mehta RL, Byrne CD. Prognostic value of the Framingham cardiovascular risk equation and the UKPDS risk engine for coronary heart disease in newly diagnosed Type 2 diabetes: Results from a United Kingdom study. Diabet Med. 2005; 22[5]:554-562. [DOI:10.1111/j.1464-5491. 2005. 01494.x].

6. Esposito K, Marfella R, Giugliano D. Plasma Interleukin18 Concentrations Are Elevated in Type 2 Diabetes [1]. Diabetes Care. 2004; 27[1]:272. [DOI: 10.2337/ diacare.27.1.272].

7. Kengne AP, Patel A, Colagiuri S, Heller S, Hamet P, Marre $\mathbf{M}$, et al. The Framingham and UK Prospective Diabetes Study [UKPDS] risk equations do not reliably estimate the probability of cardiovascular events in a large ethnically diverse sample of patients with diabetes: The Action in Diabetes and Vascular Disease: Preterax. Diabetologia. 2010; 53 [5]: 821831. [DOI: 10.1007/s00125-010-1681-4].

8. Van Hecke MV, Dekker JM, Stehouwer CD, Polak BC, Fuller JH, Sjolie AK, et al. Diabetic Retinopathy Is Associated With Mortality and Cardiovascular Disease Incidence. Diabetes Care. 2005; 19[6]:1383-1389. [DOI: 10.2337/ diacare. 28.6.1383].

9. Ikram MK, Cheung CY, Lorenzi M, Klein R, Jones TLZ, Wong TY. Retinal vascular caliber as a biomarker for diabetes microvascular complications. Diabetes Care 2013; 36[3]:750-759. [DOI: 10.2337/dc12-1554].

10. Um T, Lee DH, Kang J-W, Kim EY, Yoon YH. The degree of diabetic retinopathy in patients with type 2 diabetes correlates with the presence and severity of coronary heart disease. J Korean Med Sci. 2016; 31[8]:12921299. [DOI: 10.3346/ jkms. 2016.31. 8.1292].
11. Cheung N, Liew G, Wong TY. Current approaches to retinopathy as a predictor of cardiovascular risk. In: Hammes H-P, Porta M [eds]: Experimental Approaches to Diabetic Retinopathy. Front Diabetes. Basel, Karger, 2010, vol 20, pp 203-219. [DOI: 10.1159/000262671].

12.Jellinger PS, Handelsman $Y$, Rosenblit $P D$, Bloomgarden ZT, Fonseca VA, Garber AJ, et al. American Association of Clinical Endocrinologists and American College of Endocrinology guidelines for management of dyslipidemia and prevention of cardiovascular disease. Endocr Pract. 2017; 23:1-87. [DOI: 10.4158/ EP171764.APPGL].

13. Barkoudah E, Skali H, Uno H, Solomon SD, Pfeffer MA. Mortality rates in trials of subjects with type 2 diabetes. J Am Heart Assoc. 2012; 1[1]:e000059. [DOI: 10.1161/ JAHA. 111. 000059].

14. Cheung N, Wang JJ, Klein R, Couper DJ, Sharrett AR, Wong TY. Diabetic retinopathy and the risk of coronary heart disease: the Atherosclerosis Risk in Communities Study. Diabetes Care 2007; 30:17421746. [DOI: 10. 2337/ dc07-0264].

15. Tian PY, Jiang PC, Wang PM, Cai R, Zhang Y, He Z, et al. BMI, leisure-time physical activity, and physical fitness in adults in China: results from a series of national surveys. Lancet Diabetes Endocrinol. 2000; 4 [6]:487-497. [DOI: 10.1 016/ S2213-8587[16]000814].

16. Yang H, Young D, Gao J, Yuan Y, Shen M, Zhang Y, et al. Are blood lipids associated with microvascular complications among type 2 diabetes mellitus patients? A cross-sectional study in Shanghai, China. Lipids Health Dis. 2019; 18[1]:18. [DOI: 10.1186/ s12944- 019-0970-2].

17. Koch M, Gradaus F, Schoebel F-C, Leschke M, Grabensee B. Relevance of conventional cardiovascular risk factors for the prediction of coronary artery disease in diabetic patients on renal replacement therapy. Nephrol Dial Transplant Off Publ Eur Dial Transpl Assoc Ren Assoc. 1997;12:11871191. [DOI: 10.1093/ ndt/ 12.6.1187].

18. Williams R, Van Gaal L, Lucioni C. Assessing the impact of complications on the costs of Type II diabetes. Diabetologia. 2002; 45:S13-S17. [DOI: 10.1007/ s00125-002-0859-9].

19. Bailey CC, Sparrow JM. Co-morbidity in patients with sight-threatening diabetic retinopathy. Eye. 2001; 15:719. [DOI: 10.1038/ eye.2001.236]. 